\title{
The temporal changes of the pulsational periods of the pre-white dwarf PG 1159-035
}

\author{
J. E. S. Costa and S. O. Kepler \\ Instituto de Física, Universidade Federal do Rio Grande do Sul, 91501-970 Porto Alegre, RS, Brazil \\ e-mail:00112740@ufrgs.br
}

Received 21 November 2008 / Accepted 16 July 2008

\section{ABSTRACT}

\begin{abstract}
Context. PG 1159-035, a pre-white dwarf with $T_{\text {eff }} \simeq 140000 \mathrm{~K}$, is the prototype of the PG 1159 spectroscopic class and the DOV pulsating class. Pulsating pre-white dwarf stars evolve rapidly: the effective surface temperature decreases rapidly, the envelope contracts and the inner structure experiences stratification due to gravitational settling. These changes in the star generate variations in its oscillation periods. The measurement of temporal change in the oscillation periods, $\dot{P}$, allows us to estimate directly rates of stellar evolutionary changes, such as the cooling rate and the envelope contraction rate, providing a way to test and refine evolutionary models for pre-white dwarf pulsating stars.

Aims. Previously, only two pulsation modes of the highest amplitudes for PG 1159-035 have had their $\dot{P}$ measured: the $516.0 \mathrm{~s}$ and the 539.3 s modes. We measured the $\dot{P}$ of a larger number of pulsation modes, increasing the number of constraints for evolutionary studies of PG 1159-035. We attempted to use the secular variations in the periods of multiplets to calculate the variation in the rotational period, the envelope contraction rate, and the cooling rate of the star.

Methods. The period variations were measured directly from the PG 1159-035 observational data and refined by the $(\mathrm{O}-\mathrm{C})$ method. Results. We measured 27 pulsation mode period changes. The periods varied at rates of between 1 and $100 \mathrm{~ms} / \mathrm{yr}$, and several can be directly measured with a relative standard uncertainty below $10 \%$. For the 516.0 s mode (the highest in amplitude) in particular, not only the value of $\dot{P}$ can be measured directly with a relative standard uncertainty of $2 \%$, but the second order period change, $\ddot{P}$, can also be calculated reliably. By using the $(\mathrm{O}-\mathrm{C})$ method, we refined the $\dot{P}_{\mathrm{s}}$ and estimated the $\ddot{P}_{\mathrm{s}}$ for six other pulsation periods. As a first application, we calculated the change in the PG 1559-035 rotation period, $\dot{P}_{\text {rot }}=(-2.13 \pm 0.05) \times 10^{-6} \mathrm{ss}^{-1}$, the envelope contraction rate $\dot{R}=(-2.2 \pm 0.5) \times 10^{-13} R_{\odot} \mathrm{s}^{-1}$, and the cooling rate $\dot{T}=-1.42 \times 10^{-3} \mathrm{~K} \mathrm{~s}^{-1}$.
\end{abstract}

Key words. stars: oscillations - stars: individual: PG 1159-035 - stars: rotation - stars: white dwarfs

\section{Introduction}

PG 1159-035 is the prototype of two star classes: the PG1159 spectroscopic class and the DOV pulsating class, discovered by McGraw et al. (1979). The estimated temperature for PG 1159035 is $140000 \pm 5000 \mathrm{~K}$ (Werner et al. 1991; Dreizler et al. 1998; Jahn et al. 2007), which places this star in the pre-white dwarf sequence of the Hertzsprung-Russell diagram.

Analyzing the light curve obtained by the Whole Earth Telescope (WET) (Nather et al. 1990) in the 1989 campaign, Winget et al. (1991) (henceforth W91) identified 122 pulsation modes in PG 1159-035 with periods between 300 and $1000 \mathrm{~s}$, and spherical harmonic index $\ell=1$ or $\ell=2$. The excellent results allowed W91 to calculate the asteroseismological mass of the $\operatorname{star}\left(M / M_{\odot} \simeq 0.59\right)$ and several other stellar parameters, such as the rotation period $\left(P_{\text {rot }}=1.38 \pm 0.01\right.$ days $)$, the inclination of the rotation axis $\left(i \simeq 60^{\circ}\right)$, and a limit to the magnetic field strength $(B<6000 \mathrm{G})$. Costa et al. (2008), analyzing the PG 1159-035 combined data sets from 1983, 1985, 1989, 1993, and 2002, increased the number of identified pulsation modes to 198 , the largest after the Sun, refining the determination of the rotation period $\left(P_{\text {rot }}=1.3920 \pm 0.0008\right.$ days $)$ and other stellar parameters previously estimated by W91. The comparison of the Fourier transforms of light curves from different years indicates that the amplitudes of the pulsation modes change with time, and their intensities in the light curves can decrease to lower than the lower limit for reliable detection. Of the 198 detected modes, about $75 \%$ appear in only one of the FTs, about $25 \%$ in two or more FTs, and only 14 of the 198 pulsation modes (all of them with index $\ell=1$ ) appear in the five FTs.

About $97 \%$ of all stars end their evolution as white dwarfs. The pre-white dwarf stage is one of the "front doors" to the white dwarf cooling sequence. There are two evolutionary channels that are known to occur prior to the white dwarf stage. The first channel involves the evolution of the star from the Horizontal Branch to white dwarf by means of the Asymptotic Giant Branch (AGB), the Planetary Nebula phase, and PG1159 type stars. In the second channel, the star evolves directly from the Extended Horizontal Branch to the white dwarf stage, but without passing through the Planetary Nebula phase.

In its evolution, a pre-white dwarf star can traverse the DOV (PG1159 or GW Vir) instability strip, becoming a multiperiodic non-radial pulsating star, of periods between 100 and $1000 \mathrm{~s}$. The exact values of the pulsation periods are defined by the mass, temperature, rotation, magnetic field, and structural characteristics of the star. While it passes through the DOV/PNNV instability strip, the star cools, its envelope contracts, and its interior experiences stratification due to the gravitational settling. As a consequence, the pulsation periods change with time. The hotter the star is, the quicker are the changes in its pulsation periods. For example, in G117-B15A, a DAV white dwarf star with $T_{\text {eff }} \simeq 12000 \mathrm{~K}$, the highest amplitude period, $215 \mathrm{~s}$, changes $1 \mathrm{~s}$ 
in 8 million years, while in the hotter DOV star, PG 11559-035, with $T_{\text {eff }} \simeq 140000 \mathrm{~K}$, the $516 \mathrm{~s}$ period, the one of the highest amplitude, changes $1 \mathrm{~s}$ in only 350 years.

This work is a complementary study of the results obtained in Costa et al. (2008). We used the measured periods in the PG 1159-035 light curves of the 1983-2002 data sets to calculate the secular variation in several pulsation modes of PG 1159-035, enlarging the number of constraints for future evolutionary studies of this star. As an immediate application, we show in Sect. 4 how the measured $\dot{P}$ s in a multiplet can be used to calculate the cooling rate of the star, the envelope contraction rate and the variation in the rotational period. Part of the procedure used here was proposed by Kawaler et al. (1985a), Kawaler (1986) and other authors, but was never applied to (pre-)white dwarf stars because this requires precise period and $\dot{P}$ determinations. As highlighted in Sect. 4, the calculations are based on many hypothesis about the interior of the star, but it is a first approach taking into account the available observational data. PG 1159035 is the first and only white dwarf in which we can apply these theories and test their limitations and reliability.

\section{Measurement of secular changes in period}

\subsection{Period as a function of time}

If we assume that a pulsation period $P$ is a continuous and smooth function of time, $P=P(t)$, then it can be expanded in terms of a Taylor series,

$$
\begin{aligned}
P\left(t-t_{\mathrm{o}}\right)= & P\left(t_{\mathrm{o}}\right)+\left.\frac{\mathrm{d} P}{\mathrm{~d} t}\right|_{t=t_{\mathrm{o}}}\left(t-t_{\mathrm{o}}\right)+\left.\frac{1}{2} \frac{\mathrm{d}^{2} P}{\mathrm{~d} t^{2}}\right|_{t=t_{\mathrm{o}}}\left(t-t_{\mathrm{o}}\right)^{2} \\
& +\left.\frac{1}{6} \frac{\mathrm{d}^{3} P}{\mathrm{~d} t^{3}}\right|_{t=t_{\mathrm{o}}}\left(t-t_{\mathrm{o}}\right)^{3}+\ldots
\end{aligned}
$$

where $t_{\mathrm{o}}$ is an instant of time used as reference and $\mathrm{d}^{n} P / \mathrm{d} t^{n}$ are the period derivatives of $n$th order. In the above equation, the number of terms that must be considered depends on the values of the derivatives and the time interval $\Delta t=t-t_{\mathrm{o}}$ covered by the observational data. For DAV pulsating stars, which evolve more slowly, the terms higher than first order can be neglected, but for DOV stars (and even some DBV stars), it is necessary to consider the second order term,

$P\left(t-t_{\mathrm{o}}\right) \simeq P_{\mathrm{o}}+\dot{P}_{\mathrm{o}}\left(t-t_{\mathrm{o}}\right)+\frac{1}{2} \ddot{P}_{\mathrm{o}}\left(t-t_{\mathrm{o}}\right)^{2}$,

where $P_{\mathrm{o}}=P\left(t_{\mathrm{o}}\right)$ and $\dot{P}_{\mathrm{o}}$ and $\ddot{P}_{\mathrm{o}}$ are the first and second order derivatives, respectively, calculated at $t=t_{\mathrm{o}}$,

$\left.\dot{P}_{\mathrm{o}} \equiv \frac{\mathrm{d} P}{\mathrm{~d} t}\right|_{t=t_{\mathrm{o}}} \quad$ and

$\left.\ddot{P}_{\mathrm{o}} \equiv \frac{\mathrm{d}^{2} P}{\mathrm{~d} t^{2}}\right|_{t=t_{\mathrm{o}}}$.

\subsection{Direct measurement of $\dot{P}$}

If a period $P$ changes linearly during the time interval $\Delta t(\ddot{P} \simeq 0)$, the $\dot{P}$ accuracy, $\sigma_{\dot{P}}$, considering two measurements only, can be estimated by

$\sigma_{\dot{P}} \simeq \sqrt{2} \frac{\sigma_{P}}{\Delta t}$ where $\sigma_{P}$ is the uncertainty in the period determination. For PG 1159-035, $\sigma_{P}$ has values of between $\sim 0.002$ and $0.2 \mathrm{~s}$. For $\Delta t=19 \mathrm{yr}$, we are able to measure directly $\dot{P}$ over $\sim 3 \times 10^{-12} \mathrm{ss}^{-1}$ for the most accurately determined periods and over $\sim 1.6 \times$ $10^{-10} \mathrm{ss}^{-1}$ for the others. In other words, with the 19 -year dataset it is not possible to measure directly $\dot{P}$ below $\sim 3 \times 10^{-12} \mathrm{ss}^{-1}$, even for the most accurately measured periods. However, we can use the $(\mathrm{O}-\mathrm{C})$ method discussed in Sect. 2.3 to estimate $\dot{P}$ below this limit and improve all results obtained by direct measurement.

\subsection{The $(O-C)$ method}

The (O-C) method (see e.g. Kepler 1993) is the most wellestablished method for the measurement of secular changes in pulsating star periods. Practically, its efficiency has been demonstrated by studies of several pulsating stars, such as the DAV star G117-B15A (Kepler et al. 2005a,b) and the DBV star R548 (Mukadam et al. 2003).

By definition, the period of a periodic signal is the derivative of the time, $t$, relative to the cycle fraction (epoch), $E$ :

$$
P \equiv \frac{\mathrm{d} t}{\mathrm{~d} E}
$$

From this definition, we can derive an equation to determine the instant of time at which a maximum in the pulsation cycle occurs, $T_{\max }$, in terms of a power series of the cycle number,

$$
T_{\max }=T_{\mathrm{o}}+P_{\mathrm{o}} \cdot E+\frac{1}{2} \cdot P_{\mathrm{o}} \cdot \dot{P} \cdot E^{2}+\frac{1}{6}\left(\ddot{P} \cdot P_{\mathrm{o}}^{2}+\dot{P}^{2} \cdot P_{\mathrm{o}}\right) \cdot E^{3}+\ldots
$$

where $T_{\mathrm{o}}$ is a particular time of maximum used as temporal reference. For DAV stars, the terms over the second order in $E$ can be neglected, but in PG 1159-035 we need to consider all terms up until the third order in $E$. Equation (7) provides a powerful way of determining small $\dot{P}$ and/or $\ddot{P}$, which is known as the (O-C) method. In this method, initial values are first assumed for $T_{\mathrm{o}}$, $P_{\mathrm{o}}, \dot{P}$ and $\ddot{P}$ (we used the directly measured $\dot{P}$ and $\ddot{P}=0$ as initial values). Using the initial values and the observed values for $T_{\max }$, we then calculate the (integer) number of cycles, $E$, from Eq. (7). Using the obtained values for $E$, we finally fit Eq. (7) to the observed $T_{\max }$, recalculating the values of $T_{\mathrm{o}}, P_{\mathrm{o}}, \dot{P}$, and $\ddot{P}$. We note that the number of cycles $E$ calculated in the second step can be biased if the initial values in the first step are not sufficiently good, since generally they are not. In this case, we need to take into account the bias calculating the uncertainties in $E$ and considering all possible combinations of the numbers of cycle. For each combination, we derive a possible solution in the third step. The most likely solution is the highest probability one (fitting with lower $\chi^{2}$ ).

Even when direct measurement cannot be achieved, the period versus time plot indicates that the hypothesis stating that the period smoothly changes with time is false (assuming that the periods and their uncertainties are well determined). In this case, we cannot use the $(\mathrm{O}-\mathrm{C})$ method.

\section{Period changes in PG 1159-035}

Table 1 lists all pulsation modes detected in Fourier Transforms for data of two or more years derived by Costa et al. (2008). Dashes (-) indicate non-detected modes. The periods were calculated at the average BCT date of each data set: 2445346.87422 for $1983 ; 2446147.66421$ for $1985 ; 2447593.33756$ for 1989 ; 2449065.92947 for 1993; and 2452410.63535 for 2002 . The 
Table 1. Observed periods (in seconds) in each yearly data set.

\begin{tabular}{|c|c|c|c|c|c|c|c|c|}
\hline $\begin{array}{c}\text { Mode } \\
\text { (s) }\end{array}$ & $\overline{\bar{\ell}}$ & $\bar{m}$ & $\overline{k k \pm 1}$ & $\overline{1983}$ & $\overline{1985}$ & 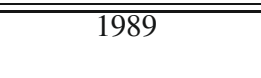 & 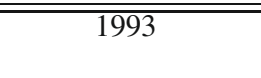 & 2002 \\
\hline 376.0 & 2 & 0 & 22 & $376.6472 \pm 0.0019$ & $376.0411 \pm 0.0057$ & - & - & - \\
\hline 387.4 & 2 & 0 & 27 & - & - & $387.4972 \pm 0.0164$ & _- & $387.4878 \pm 0.0160$ \\
\hline 390.3 & 1 & -1 & 14 & $390.2763 \pm 0.0009$ & - & $390.2959 \pm 0.0050$ & - & $390.3486 \pm 0.0316$ \\
\hline 390.8 & 1 & 0 & 14 & - & - & - & $390.7740 \pm 0.0152$ & $390.9327 \pm 0.0476$ \\
\hline 397.2 & 2 & +2 & 28 & - & $397.2251 \pm 0.0042$ & - & - & $397.2591 \pm 0.0226$ \\
\hline 399.0 & 2 & +1 & 28 & - & - & $398.9141 \pm 0.0140$ & - & $399.1425 \pm 0.0112$ \\
\hline 400.0 & 2 & 0 & 28 & - & - & $400.0585 \pm 0.0039$ & $400.0396 \pm 0.0057$ & $400.0383 \pm 0.0121$ \\
\hline 401.6 & 2 & -2 & 28 & - & - & - & - & $401.1103 \pm 0.0201$ \\
\hline 412.0 & 1 & 0 & 15 & - & - & $412.0097 \pm 0.0093$ & - & $412.0451 \pm 0.0122$ \\
\hline 414.3 & 2 & -1 & 28 & - & - & $414.3894 \pm 0.0093$ & $414.4157 \pm 0.0086$ & $414.3356 \pm 0.0114$ \\
\hline 415.5 & 2 & -2 & 28 & - & $415.4274 \pm 0.0030$ & $415.6016 \pm 0.0050$ & - & $415.7585 \pm 0.0155$ \\
\hline 422.5 & 2 & +2 & 30 & $422.5397 \pm 0.0008$ & - & - & $422.5630 \pm 0.0027$ & $422.5768 \pm 0.0056$ \\
\hline 426.2 & 2 & -1 & 30 & - & - & - & $426.2398 \pm 0.0064$ & $426.3075 \pm 0.0089$ \\
\hline 427.5 & 2 & -2 & 30 & - & - & $427.5585 \pm 0.0033$ & $427.5125 \pm 0.0060$ & $427.4328 \pm 0.0514$ \\
\hline 430.3 & 1 & +1 & 16 & $430.2347 \pm 0.0032$ & - & - & - & $430.3800 \pm 0.0407$ \\
\hline 434.2 & 1 & -1 & 16 & - & $434.3305 \pm 0.0030$ & - & - & $434.1443 \pm 0.0179$ \\
\hline 436.5 & 2 & +1 & 31 & - & - & $436.5258 \pm 0.0160$ & $436.5766 \pm 0.0074$ & $436.5366 \pm 0.0226$ \\
\hline 439.2 & 2 & -1 & 31 & - & - & $439.2814 \pm 0.0134$ & $439.2268 \pm 0.0089$ & $439.2376 \pm 0.0149$ \\
\hline 440.6 & 2 & -2 & 31 & - & - & $440.6920 \pm 0.0061$ & $440.6430 \pm 0.0043$ & $440.4827 \pm 0.0124$ \\
\hline 451.5 & 1 & +1 & 17 & $451.5928 \pm 0.0005$ & $451.5776 \pm 0.0012$ & $451.5906 \pm 0.0019$ & $451.6007 \pm 0.0015$ & $451.5806 \pm 0.0030$ \\
\hline 452.4 & 1 & 0 & 17 & $452.4275 \pm 0.0008$ & $452.3713 \pm 0.0022$ & $452.4310 \pm 0.0021$ & $452.4324 \pm 0.0022$ & $452.4478 \pm 0.0035$ \\
\hline 453.2 & 1 & -1 & 17 & $453.2534 \pm 0.0016$ & - & - & $453.2800 \pm 0.0039$ & $453.2743 \pm 0.0066$ \\
\hline 493.7 & 1 & +1 & 19 & $493.7640 \pm 0.0005$ & $493.7490 \pm 0.0013$ & $493.7984 \pm 0.0024$ & $493.7941 \pm 0.0025$ & - \\
\hline 494.8 & 1 & 0 & 19 & $494.9043 \pm 0.0012$ & - & $494.8633 \pm 0.0178$ & $494.8046 \pm 0.0094$ & - \\
\hline 511.9 & 2 & 0 & 37 & - & - & $512.0018 \pm 0.0293$ & $511.9741 \pm 0.0123$ & - \\
\hline 515.0 & 2 & -1 & 37 & $515.0718 \pm 0.0031$ & $515.0311 \pm 0.0033$ & - & $515.0300 \pm 0.0140$ & $514.9390 \pm 0.0194$ \\
\hline 516.0 & 1 & +1 & 20 & $516.0260 \pm 0.0004$ & $516.0374 \pm 0.0006$ & $516.0548 \pm 0.0016$ & $516.0663 \pm 0.0014$ & $516.1028 \pm 0.0019$ \\
\hline 517.1 & 1 & 0 & 20 & $517.1225 \pm 0.0023$ & $517.1402 \pm 0.0014$ & $517.1663 \pm 0.0024$ & $517.1827 \pm 0.0020$ & - \\
\hline 518.2 & 1 & -1 & 20 & - & - & $518.2884 \pm 0.0028$ & $518.2960 \pm 0.0027$ & $518.2980 \pm 0.0077$ \\
\hline 526.4 & 2 & -1 & 38 & - & - & $526.3592 \pm 0.0566$ & - & $526.4515 \pm 0.0165$ \\
\hline 536.9 & 1 & +1 & 21 & $536.8412 \pm 0.0031$ & - & $536.9530 \pm 0.0075$ & $536.8223 \pm 0.0155$ & $537.0081 \pm 0.0127$ \\
\hline 538.1 & 1 & 0 & 21 & $538.1585 \pm 0.0008$ & - & $538.1547 \pm 0.0026$ & - & $538.1696 \pm 0.0047$ \\
\hline 539.3 & 1 & -1 & 21 & $539.3593 \pm 0.0006$ & $539.4030 \pm 0.0010$ & $539.3552 \pm 0.0020$ & $539.3546 \pm 0.0012$ & - \\
\hline 540.9 & 2 & -2 & 39 & - & - & - & $540.9620 \pm 0.0150$ & $540.8784 \pm 0.0600$ \\
\hline 544.3 & 2 & +2 & 40 & - & - & - & $544.3258 \pm 0.0204$ & $544.3305 \pm 0.0235$ \\
\hline 557.1 & 1 & +1 & 22 & - & - & $557.1386 \pm 0.0034$ & $557.1137 \pm 0.0138$ & $557.1383 \pm 0.0139$ \\
\hline 558.4 & 1 & 0 & 22 & - & - & $558.4476 \pm 0.0043$ & $558.4449 \pm 0.0073$ & $558.4297 \pm 0.0066$ \\
\hline 559.7 & 1 & -1 & 22 & - & - & $559.7135 \pm 0.0124$ & $559.7608 \pm 0.0078$ & $559.7461 \pm 0.0258$ \\
\hline 561.9 & 2 & 0 & 41 & $561.9714 \pm 0.0028$ & - & - & $562.0109 \pm 0.0226$ & $561.7732 \pm 0.0261$ \\
\hline 641.4 & 1 & 0 & 26 & - & - & $641.5343 \pm 0.0138$ & $641.4278 \pm 0.0329$ & - \\
\hline 644.9 & 1 & -1 & 26 & - & $644.8953 \pm 0.0043$ & $644.9868 \pm 0.0165$ & - & - \\
\hline 668.5 & 1 & 0 & 27 & - & - & $668.5441 \pm 0.0446$ & - & $668.4789 \pm 0.0590$ \\
\hline 685.8 & 1 & +1 & 28 & - & - & $685.7817 \pm 0.0899$ & $685.8593 \pm 0.0337$ & - \\
\hline 689.7 & 1 & -1 & 28 & - & - & $689.7606 \pm 0.0321$ & $689.8163 \pm 0.0234$ & - \\
\hline 705.8 & 1 & +1 & 29 & - & $705.8474 \pm 0.0094$ & $705.9310 \pm 0.0220$ & - & - \\
\hline 727.0 & 1 & +1 & 30 & - & - & $727.1008 \pm 0.0250$ & - & $727.0057 \pm 0.0425$ \\
\hline 729.6 & 1 & 0 & 30 & - & - & $729.5007 \pm 0.0614$ & $729.7205 \pm 0.0409$ & - \\
\hline 731.6 & 1 & -1 & 30 & - & - & $731.4527 \pm 0.0187$ & $731.6075 \pm 0.0280$ & $731.7440 \pm 0.0706$ \\
\hline 755.3 & 1 & -1 & 31 & - & - & $755.3732 \pm 0.0635$ & $755.2315 \pm 0.0489$ & - \\
\hline 812.5 & 1 & +1 & 34 & - & $812.4437 \pm 0.0124$ & $812.5725 \pm 0.0537$ & - & - \\
\hline 819.7 & 2 & -2 & 60 & $819.7720 \pm 0.0049$ & - & $819.9484 \pm 0.0277$ & $819.5586 \pm 0.0289$ & - \\
\hline 842.8 & 1 & -1 & 35 & $842.8731 \pm 0.0032$ & - & $842.8873 \pm 0.0258$ & - & - \\
\hline 861.7 & 1 & 0 & 36 & - & $861.6775 \pm 0.0092$ & - & $861.8524 \pm 0.0293$ & - \\
\hline 877.6 & 1 & +1 & 37 & - & - & - & $877.6142 \pm 0.0703$ & $877.7300 \pm 0.0660$ \\
\hline
\end{tabular}

period uncertainties, $\sigma_{\mathrm{P}}$, were calculated from nonlinear fitting of sinusoidal curves for all the detected frequencies, using the Levenberg-Marquardt method. Monte Carlo simulations of synthetic light curves for PG 1159-035, as proposed by Costa \& Kepler (2000), indicate that the calculated uncertainties in period (and also in phase and amplitude) are excellent when the pulsation frequencies are solved and the amplitudes are constant. However, the calculated uncertainties can be underestimated for modes with amplitudes that change during the observations or for non-solved frequencies.

\subsection{Direct measurement}

Figure 2 shows the period (in seconds) versus time (in years) plots for all the modes detected in three or more FTs. Above 
each graph, there is the average period (in seconds) and the ( $\ell, m$, $k$ ) indices of the pulsation mode (the $k$ index has an uncertainty \pm 2 ). The error bars represent the $1 \sigma$ uncertainties in the period measurement. The slope of the fitted curve is indicated at the top of each graph.

In most cases, the points distribution is consistent statistically with a linear fit, taking into account the uncertainties in the period measurements. This is particularly notable in the cases of the $422.5 \mathrm{~s}, 427.5 \mathrm{~s}, 440.6 \mathrm{~s}, 494.8 \mathrm{~s}, 516.0 \mathrm{~s}$, and $517.1 \mathrm{~s}$ pulsation modes. In the $451.5 \mathrm{~s}, 493.7 \mathrm{~s}, 536.9 \mathrm{~s}$, and $819.7 \mathrm{~s}$ plots, the points are scattered with large dispersions relative to the straight lines. It is possible that some points with lower confidence levels have been mistakenly identified. The measured $\dot{P}$ for the pulsation modes in Fig. 2 are listed in Table 2. The relative standard uncertainty $\left(\sigma_{\dot{P}} /|\dot{P}|\right)$ in the $\dot{P}$ measurement of the 516.0 s period is only $2.0 \%$ and for other five periods the relative standard uncertainties are less than $10 \%: 422.5 \mathrm{~s}, 440.6 \mathrm{~s}, 452.4 \mathrm{~s}, 453.2 \mathrm{~s}$, and $517.1 \mathrm{~s}$.

\section{2. (O-C) method}

We used the directly measured $\dot{P}$ as initial values (setting $\ddot{P}=0$ ) in the (O-C) method to refine the $\dot{P}$ and periods of the modes present in three or more FTs. With three points only, the fitting provides preliminary values for future calculations. We also used the $(\mathrm{O}-\mathrm{C})$ method to calculate $\ddot{P}$ of the modes present in four or five of the FTs. Our most accurate results are given in Table 2.

The accuracy of the $\dot{P}$ determination is, in general, two orders of magnitude smaller than that of direct measurement. We note that the $(\mathrm{O}-\mathrm{C})$ fitting assumes that the periods change smoothly with time. If the changes are not smooth or if they depend on higher order terms, the $(\mathrm{O}-\mathrm{C})$ can produce incorrect results. It is also important to note that although the most suitable solution is the most probable one, it is not necessarily the true solution and no solution has an uncertainty of below $1 \%$. Future photometric observations of PG 1159-035 should confirm or discard our results.

We comment on three particular cases: changes in the $516.0 \mathrm{~s}, 517.1 \mathrm{~s}$, and $539.1 \mathrm{~s}$ periods. The two first cases were identified by W91 and Costa et al. (2008) as the $m=+1$ and $m=0$ components, respectively, of the triplet $(\ell=1)$ with radial index $k=20 \pm 2$; they are used in Sect. 4 to calculate PG 1159035 evolutionary rates. The $516.0 \mathrm{~s}$ mode is clearly present in the FT of all data sets. The same occurs with the $517.1 \mathrm{~s}$ mode, apart from the 2002 FT. The $539.1 \mathrm{~s}$ period was identified as the component $m=-1$ of the $k=21 \pm 2$ triplet and was the second period to have its $\dot{P}$ determined (Costa et al. 1995).

\subsection{The $516.0 \mathrm{~s}$ mode}

The $516.0 \mathrm{~s}$ pulsation mode $(\ell=1, m=+1, k=20 \pm 2)$ is one of the highest amplitude modes of PG 1159-035. Using the 1983, 1985, 1989, and 1993 data sets, Costa et al. (1999) achieved the first direct measurement of period change in a (pre-)white dwarf, deriving $\dot{P}_{516}=(+13.0 \pm 2.6) \times 10^{-11} \mathrm{ss}^{-1}$. They refined this value with the (second order) $(\mathrm{O}-\mathrm{C})$ method finding $\dot{P}_{516}=(+13.07 \pm$ $0.03) \times 10^{-11} \mathrm{ss}^{-1}$

Fitting the curve $P=P_{\mathrm{o}}+\dot{P}\left(t-T_{\mathrm{o}}\right)$ to the data in Table 1 , we measure $P_{\mathrm{o}}=516.0516 \pm 0.0005 \mathrm{~s}$ and $\dot{P}=(+12.8 \pm 0.3) \times$ $10^{-11} \mathrm{ss}^{-1}$ at $T_{\mathrm{o}}=2447593.334592(\mathrm{BCT})$. Including $\ddot{P}$ in the fitting, $P\left(T-T_{\mathrm{o}}\right)=P_{\mathrm{o}}+\dot{P} \cdot\left(T-T_{\mathrm{o}}\right)+1 / 2 \cdot \ddot{P} \cdot\left(T-T_{\mathrm{o}}\right)^{2}$, we obtain $P_{\mathrm{o}}=516.0534 \pm 0.0008 \mathrm{~s}, \dot{P}=(+13.2 \pm 0.3) \times 10^{-11} \mathrm{ss}^{-1}$ and $\ddot{P}=(-7.8 \pm 0.7) \times 10^{-20} \mathrm{ss}^{-2}$, at the same $T_{\mathrm{o}}$. The two fitted

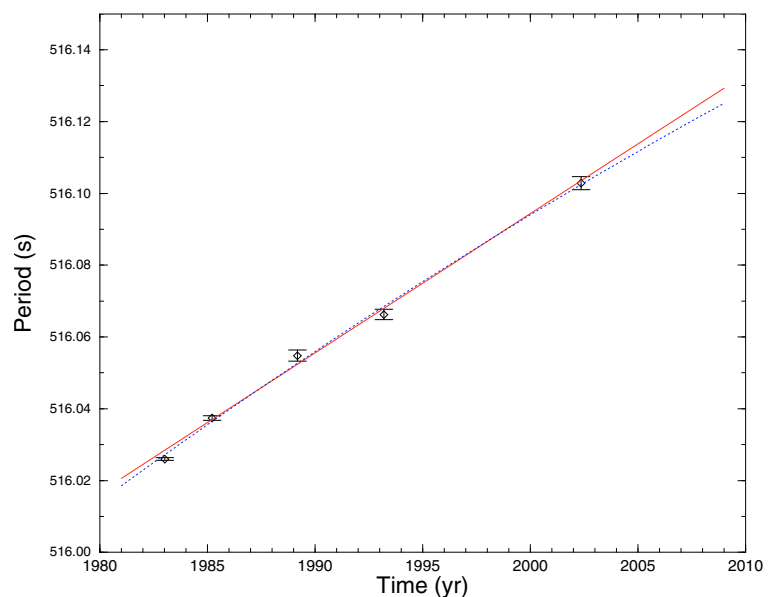

Fig. 1. Temporal change in the $516.0 \mathrm{~s}$ pulsation period. The solid line represents a linear fitting and the dashed line a quadratic fitting.

curves are shown in Fig. 1. Using these results as initial values in the (third order) $(\mathrm{O}-\mathrm{C})$ method, we find a best-fit solution with the parameters $T_{\mathrm{o}}=2447593.93114 \pm 0.00002(\mathrm{BCT}), P_{\mathrm{o}}=$ $516.065545 \pm 0.00001 \mathrm{~s}, \dot{P}=(+13.146 \pm 0.003) \times 10^{-11} \mathrm{ss}^{-1}$, and $\ddot{P}_{\mathrm{o}}=(1.93 \pm 0.08) \times 10^{-20} \mathrm{ss}^{-1}$. With the introduction of the third order term, the new (O-C) result for $\dot{P}$ differs by a margin of approximately $2.5 \sigma$ from the previous value calculated by Costa et al. (1999).

\subsection{The $517.1 \mathrm{~s}$ mode}

The $517.1 \mathrm{~s}$ period was identified as the central peak $(m=0)$ of the $k=20 \pm 2$ triplet, where the $518.2 \mathrm{~s}$ and $516.0 \mathrm{~s}$ modes are the $m=-1$ and $m=+1$ components, respectively. This mode appears in all the FTs, apart from the 2002 FT. By fitting a (linear) curve to the data in Table 1, we obtain $\dot{P}=(+18.2 \pm 0.8) \times 10^{-11} \mathrm{ss}^{-1}$; using this value as the initial value in the third order $(\mathrm{O}-\mathrm{C})$ fitting, we determine as the best-fit solution $T_{\mathrm{o}}=2447593.33920 \pm 0.00003$ (BCT), $P_{\mathrm{o}}=517.16755 \pm 0.00009 \mathrm{~s}, \dot{P}_{\mathrm{o}}=(+15.172 \pm 0.045) \times 10^{-11} \mathrm{ss}^{-1}$, and $\ddot{P}_{\mathrm{o}}=(-81.7 \pm 2.7) \times 10^{-20} \mathrm{ss}^{-1}$. The $(\mathrm{O}-\mathrm{C})$ results differs by $\sim 3.7 \sigma$ from the directly measured one. This difference is, at least partially, due to introduction of the third order term.

\subsection{The $539.3 \mathrm{~s}$ mode}

The $539.3 \mathrm{~s}$ pulsation mode, identified $\ell=1, m=-1$, and $k=21 \pm 2$ was detected in the 1983, 1989, and 1993 FTs only and was the second period for which $\dot{P}$ was measured. Costa et al. (1995) used the (O-C) method applied to the same data sets to calculate its $\dot{P}$, obtaining $\dot{P}=(-0.82 \pm 0.04) \times 10^{-11} \mathrm{ss}^{-1}$. Using the same data sets but with an improved data reduction process, and frequency determination we obtained $\dot{P}=$ $(-1.5 \pm 0.4) \times 10^{-11} \mathrm{ss}^{-1}$ from the direct measurement and $\dot{P}=$ $(-0.339 \pm 0.015) \times 10^{-11} \mathrm{ss}^{-1}, T_{\mathrm{o}}=2447593.33895 \pm 0.00002$ (BCT), and $P_{\mathrm{o}}=539.35572 \pm 0.00001 \mathrm{~s}$ from the $(\mathrm{O}-\mathrm{C})$ fitting. The large difference between the present $(\mathrm{O}-\mathrm{C})$ result and the $(\mathrm{O}-\mathrm{C})$ result obtained by Costa et al. (1995) is because in this previous work the times of maximum and their uncertainties were calculated using a linear fitting by a single sinusoidal curve. As demonstrated by Costa et al. (1999) and Costa \& Kepler (2000), the linear fitting does not take into account the interference of the other pulsation modes over the fitted mode and underestimate the calculated uncertainties. 

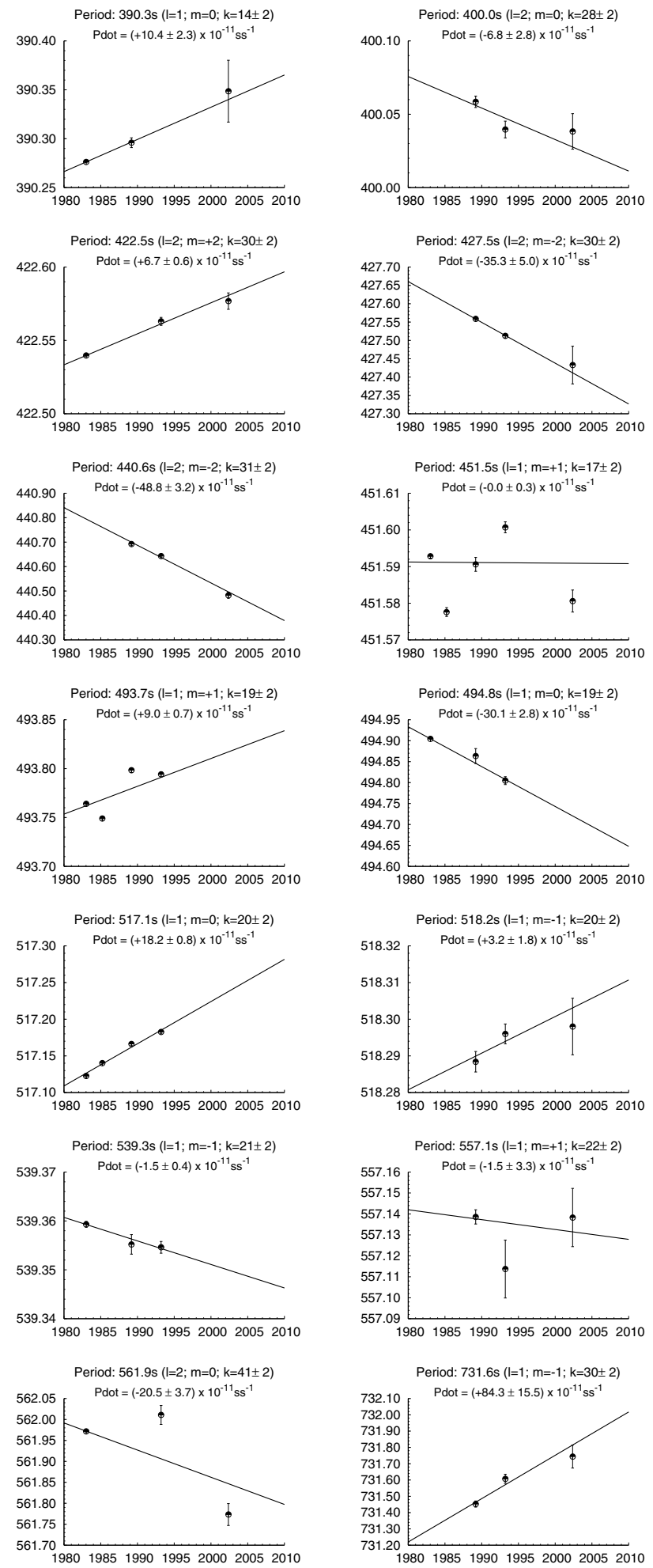
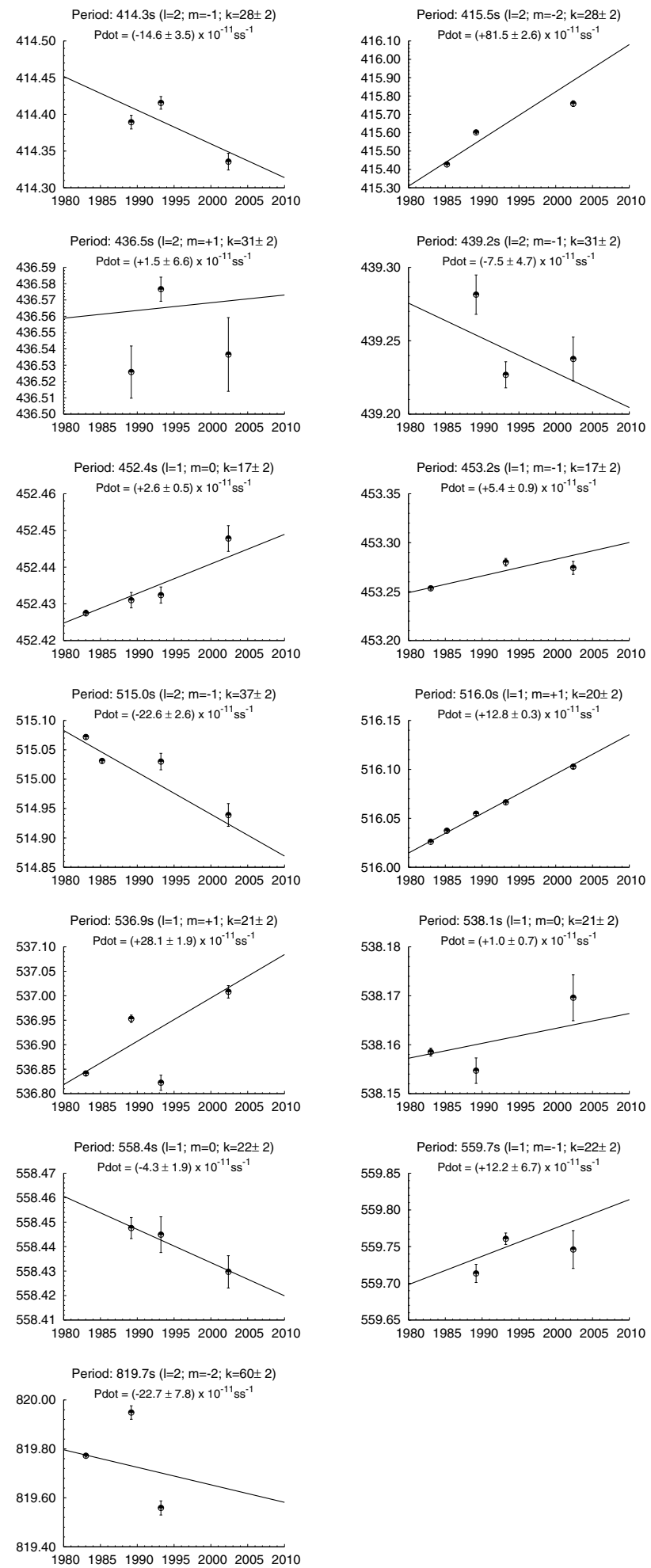

Fig. 2. Temporal changing in the periods of 27 PG 1159-035 pulsation modes. The vertical axis of each graph represents the periods, in seconds, and the horizontal axis is the time, in years. The bars represent the $\pm 1 \sigma$ uncertainties. The mode identification (period in seconds and the $\ell, m$ and $k$ indices) appears in the first line above each graph. In the second line "Pdot" $(\dot{P})$ is the slope of the straight line fitted to the points.

\section{Application: calculating evolutionary timescales}

\subsection{Variation in the stellar rotation period}

The observed frequency spacings between the $m \neq 0$ components and the central $(m=0)$ peak of a multiplet are caused by a combination of the effect of the stellar rotation and the effect of the magnetic field of the star over the observed pulsation frequencies (see e.g. Jones et al. 1989):

$\delta v_{m}=v_{m}-v_{0}=\delta v_{\mathrm{rot}, m}+\delta v_{\mathrm{mag}, m}$

To a first-order approximation, the rotation splitting $\delta v_{\mathrm{rot}, m}$ is proportional to the angular rotation frequency $\Omega_{\text {rot }}$ multiplied 
Table 2. Results from the direct method and (O-C) fitting.

\begin{tabular}{|c|c|c|c|c|c|c|c|c|c|}
\hline \multicolumn{4}{|c|}{ Pulsation mode } & \multicolumn{2}{|r|}{ Direct } & \multicolumn{4}{|c|}{ (O-C) Fitting } \\
\hline $\begin{array}{l}\langle P\rangle \\
(\mathrm{s})\end{array}$ & $\ell$ & $m$ & $\begin{array}{c}k \\
\pm 2\end{array}$ & $N$ & $\begin{array}{c}\dot{P} \\
\times 10^{-11} \mathrm{ss}^{-1}\end{array}$ & $\begin{array}{c}T_{\mathrm{o}} \\
(\mathrm{BCT})\end{array}$ & $\begin{array}{l}P_{\mathrm{o}} \\
(\mathrm{s})\end{array}$ & $\begin{array}{c}\dot{P} \\
\times 10^{-11} \mathrm{Ss}^{-1}\end{array}$ & $\begin{array}{c}\ddot{P} \\
\times 10^{-19} \mathrm{ss}^{-2}\end{array}$ \\
\hline 390.3 & 1 & 0 & 14 & 3 & $+10.4 \pm 2.3$ & $2447593.33753 \pm 0.00009$ & $390.30088 \pm 0.00003$ & $12.596 \pm 0.020$ & \\
\hline 400.0 & 2 & 0 & 28 & 3 & $-6.8 \pm 2.8$ & $2449065.92791 \pm 0.00012$ & $400.04271 \pm 0.00002$ & $-0.123 \pm 0.027$ & \\
\hline 414.3 & 2 & -1 & 28 & 3 & $-14.6 \pm 3.5$ & $2449065.92697 \pm 0.00018$ & $414.41413 \pm 0.00004$ & $-22.065 \pm 0.042$ & \\
\hline 415.5 & 2 & -2 & 28 & 3 & $+81.5 \pm 2.6$ & $2447593.33537 \pm 0.00008$ & $415.60748 \pm 0.00008$ & $55.633 \pm 0.042$ & \\
\hline 422.5 & 2 & +2 & 30 & 3 & $+6.7 \pm 0.6$ & $2449065.92752 \pm 0.00006$ & $422.56237 \pm 0.00001$ & $4.984 \pm 0.008$ & \\
\hline 427.5 & 2 & -2 & 30 & 3 & $-35.3 \pm 5.0$ & $2449065.93108 \pm 0.00012$ & $427.51584 \pm 0.00004$ & $-33.956 \pm 0.060$ & \\
\hline 436.5 & 2 & +1 & 31 & 3 & $+1.5 \pm 6.6$ & $2449065.92902 \pm 0.00015$ & $436.59526 \pm 0.00006$ & $-10.899 \pm 0.052$ & \\
\hline 439.2 & 2 & -1 & 31 & 3 & $-7.5 \pm 4.7$ & $2449065.92774 \pm 0.00017$ & $439.23003 \pm 0.00005$ & $-9.066 \pm 0.048$ & \\
\hline 440.6 & 2 & -2 & 31 & 3 & $-48.8 \pm 3.2$ & $2449065.92750 \pm 0.00008$ & $440.64399 \pm 0.00002$ & $-47.992 \pm 0.025$ & \\
\hline 451.5 & 1 & +1 & 17 & 5 & $0.0 \pm 0.3$ & $2449065.92845 \pm 0.00003$ & $451.60156 \pm 0.00001$ & $-1.498 \pm 0.005$ & $-2.908 \pm 0.001$ \\
\hline 452.4 & 1 & 0 & 17 & 4 & $+2.6 \pm 0.5$ & $2449065.93009 \pm 0.00004$ & $452.43494 \pm 0.00002$ & $4.720 \pm 0.007$ & $-0.333 \pm 0.014$ \\
\hline 453.2 & 1 & -1 & 17 & 3 & $+5.4 \pm 0.9$ & $2449065.92972 \pm 0.00007$ & $453.28156 \pm 0.00002$ & $4.143 \pm 0.013$ & \\
\hline 493.7 & 1 & +1 & 19 & 4 & $+9.0 \pm 0.7$ & $2447593.33963 \pm 0.00003$ & $493.79587 \pm 0.00007$ & $15.102 \pm 0.041$ & $8.656 \pm 0.173$ \\
\hline 494.8 & 1 & 0 & 19 & 3 & $-30.1 \pm 2.8$ & $2447593.33761 \pm 0.00024$ & $494.85869 \pm 0.00005$ & $-30.526 \pm 0.093$ & \\
\hline 515.0 & 2 & -1 & 31 & 4 & $-22.6 \pm 2.6$ & $2449065.93209 \pm 0.00024$ & $515.00799 \pm 0.00018$ & $-15.661 \pm 0.033$ & $6.332 \pm 0.130$ \\
\hline 516.0 & 1 & +1 & 20 & 5 & $+12.8 \pm 0.3$ & $2449065.93114 \pm 0.00002$ & $516.06545 \pm 0.00001$ & $13.146 \pm 0.003$ & $0.193 \pm 0.008$ \\
\hline 517.1 & 1 & 0 & 20 & 4 & $+18.2 \pm 0.8$ & $2447593.33920 \pm 0.00003$ & $517.16755 \pm 0.00009$ & $15.172 \pm 0.045$ & $-8.166 \pm 0.273$ \\
\hline 518.2 & 1 & -1 & 20 & 3 & $+3.2 \pm 1.8$ & $2449065.93082 \pm 0.00005$ & $518.29767 \pm 0.00001$ & $-0.255 \pm 0.016$ & \\
\hline 536.9 & 1 & +1 & 21 & 4 & $+28.1 \pm 1.9$ & $2449065.93110 \pm 0.00025$ & $536.82344 \pm 0.00010$ & $30.771 \pm 0.037$ & $6.110 \pm 0.071$ \\
\hline 538.1 & 1 & 0 & 21 & 3 & $+1.0 \pm 0.7$ & $2447593.33642 \pm 0.00003$ & $538.15390 \pm 0.00002$ & $4.304 \pm 0.010$ & \\
\hline 539.3 & 1 & -1 & 21 & 3 & $-1.5 \pm 0.4$ & $2447593.33895 \pm 0.00002$ & $539.35572 \pm 0.00001$ & $-0.339 \pm 0.015$ & \\
\hline 557.1 & 1 & +1 & 22 & 3 & $-1.5 \pm 3.3$ & $2449065.92978 \pm 0.00021$ & $557.11218 \pm 0.00005$ & $-3.419 \pm 0.057$ & \\
\hline 558.4 & 1 & 0 & 22 & 3 & $-4.3 \pm 1.9$ & $2449065.92676 \pm 0.00011$ & $558.44566 \pm 0.00003$ & $-10.946 \pm 0.031$ & \\
\hline 559.7 & 1 & -1 & 22 & 3 & $+12.2 \pm 6.7$ & $2449065.92945 \pm 0.00012$ & $559.76600 \pm 0.00005$ & $11.602 \pm 0.050$ & \\
\hline 561.9 & 2 & 0 & 41 & 3 & $-20.5 \pm 3.7$ & $2449065.93013 \pm 0.00034$ & $562.00642 \pm 0.00004$ & $-19.230 \pm 0.044$ & \\
\hline 731.6 & 1 & -1 & 30 & 3 & $+84.3 \pm 15$ & $2449065.92821 \pm 0.00033$ & $731.61198 \pm 0.00012$ & $88.859 \pm 0.142$ & \\
\hline 819.7 & 2 & -2 & 60 & 3 & $-22.7 \pm 7.8$ & $2447593.34294 \pm 0.00023$ & $819.96585 \pm 0.00013$ & $-40.498 \pm 0.195$ & \\
\hline
\end{tabular}

by $m$, while the magnetic splitting $\delta v_{\text {mag }}$ is proportional to the magnetic strength $B=|\boldsymbol{B}|$ multiplied by $m^{2}$ (see e.g. W91),

$\delta v_{m} \simeq m C \Omega_{\mathrm{rot}}+m^{2} D B^{2}$,

where $C$ and $D$ are constants. Costa et al. (2008) demonstrated that the PG 1159-035 magnetic strength is weak, $B<2000 \mathrm{G}$, and that the contribution of the average magnetic splitting in the total observed splitting is lower than $1 \%\left(\delta v_{\text {mag,m }}=0.007 \pm\right.$ $0.002 \mu \mathrm{Hz}$ ), which allows us to approximate Eq. (9) with:

$\delta v_{m} \simeq m C \Omega_{\mathrm{rot}}$.

The proportionality constant $C$ can be rewritten (Hansen et al. 1977) as

$C=1-C_{0}-C_{1}$,

where $C_{0}$ is the uniform rotation coefficient that depends on $k$ and $\ell$, and equals $C_{0}=C_{0}(k, \ell)$, and $C_{1}$, related to nonuniform rotation effects, is a function of $k, \ell$ and $|m|$, and equals $C_{1}=C_{1}(k, \ell,|m|)$. The second coefficient depends on the adiabatic pulsation properties, equilibrium structure, and rotation law. If we assume uniform rotation, $C_{1}=0$ and then $C \simeq 1-C_{0}$. In the asymptotic limit with high radial overtones $(k \gg 1)$, the uniform rotation coefficient can be approximated by (Brickhill 1975) $C_{0} \simeq[\ell(\ell+1)]^{-1}$. For $\ell=1$ modes, $C_{0} \simeq 1 / 2, C \simeq 1 / 2$, and Eq. (10) can be rewritten as:

$v_{m}-v_{0} \simeq m \frac{1}{2} \Omega_{\mathrm{rot}}$.

This approximation is accurate to about $5 \%$ for the PG 1159-035 frequency splittings (Costa et al. 2008).
Deriving both sides of the equation above relative to time,

$\dot{v}_{m}-\dot{v}_{0} \simeq m \frac{1}{2} \dot{\Omega}_{\text {rot }}$

or in terms of periods,

$\dot{P}_{\text {rot }} \simeq \frac{2}{m}\left(\frac{\dot{P}_{m}}{P_{m}^{2}}-\frac{\dot{P}_{0}}{P_{0}^{2}}\right) P_{\text {rot }}^{2}$,

where $\dot{P}_{\text {rot }}$ is the instantaneous rotation period change rate.

Taking the time derivative of Eq. (14) we obtain the second derivative $\ddot{P}_{\text {rot }}$ :

$$
\begin{aligned}
\ddot{P}_{\text {rot }} \simeq & -\frac{4}{m}\left(\frac{\dot{P}_{m}^{2}}{P_{m}^{3}}-\frac{\dot{P}_{0}^{2}}{P_{0}^{3}}\right) P_{\text {rot }}^{2}+\frac{2}{m}\left(\frac{\ddot{P}_{m}}{P_{m}^{2}}-\frac{\ddot{P}_{0}}{P_{0}^{2}}\right) P_{\text {rot }}^{2} \\
& +\frac{4}{m}\left(\frac{\dot{P}_{m}}{P_{m}^{2}}-\frac{\dot{P}_{0}}{P_{0}^{2}}\right) P_{\text {rot }} \dot{P}_{\text {rot }} .
\end{aligned}
$$

In Eq. (16), the second term is the dominant term for PG 1159035 and the other two terms can be neglected:

$$
\ddot{P}_{\text {rot }} \simeq \frac{2}{m}\left(\frac{\ddot{P}_{m}}{P_{m}^{2}}-\frac{\ddot{P}_{0}}{P_{0}^{2}}\right) P_{\text {rot }}^{2} .
$$

We note that with at least two well-determined $\dot{P}$ in a triplet, we are able to calculate the rotation period change rate, $\dot{P}_{\text {rot }}$, using Eq. (14). If $\ddot{P}_{0}$ and $\ddot{P}_{m}$ are both known, we can also calculate $\ddot{P}_{\text {rot }}$ from Eq. (16).

Using the $(\mathrm{O}-\mathrm{C})$ method results for the $(m=0) 517.1 \mathrm{~s}$ and the $(m=+1) 516.0 \mathrm{~s}$ modes and the rotation period, 
$P_{\text {rot }}=1.3920 \pm 0.0008$ days (Costa et al. 2008), in Eqs. (14) and (16), we obtain:

$\dot{P}_{\text {rot }} \simeq(-2.13 \pm 0.05) \times 10^{-6} \mathrm{ss}^{-1}$ and

$\ddot{P}_{\text {rot }} \simeq(+9.0 \pm 0.3) \times 10^{-14} \mathrm{ss}^{-2}$.

Using the direct method results, the rates are $\dot{P}_{\text {rot }} \simeq(-2.5 \pm 0.3) \times$ $10^{-6} \mathrm{ss}^{-1}$ and $\ddot{P}_{\text {rot }} \simeq(+8.0 \pm 8.8) \times 10^{-14} \mathrm{ss}^{-2}$.

The accuracy of $\dot{P}_{\text {rot }}$ measured by Eq. (14) depends strongly on the accuracies of $\dot{P}_{m}$ and $\dot{P}_{0}$, far more than on the accuracies of the pulsation periods or rotation period. Keeping unchanged $\sigma_{\dot{P}_{0}}$ and $\sigma_{\dot{P}_{m}}$ and increasing the uncertainties in $P_{0}$ and $P_{m}$ by a factor of 10 , the $\dot{P}_{\text {rot }}$ uncertainty is almost unaffected, although it changes sensitively with $\sigma_{\dot{P}_{0}}$ and $\sigma_{\dot{P}_{m}}$. However, the uncertainties in the period determination, $\sigma_{P_{m}}$, affect indirectly the $\dot{P}_{\text {rot }}$ uncertainty, because both $\sigma_{\dot{P}_{0}}$ and $\sigma_{\dot{P}_{m}}$ depend on $\sigma_{P_{m}}$ and $\sigma_{P_{0}}$. If the period uncertainty are then underestimated, the uncertainty in $\dot{P}_{\text {rot }}$ must also be. The results above and the following results are discussed in the final section of this article.

\subsection{The contraction rate}

We now calculate the contraction timescale, $R / \dot{R}$, and contraction rate, $\dot{R}$. For a star of uniform rotation and negligible mass loss (Kawaler 1986; Costa et al. 1999),

$\frac{\dot{R}}{R} \simeq \frac{1}{2} \frac{\dot{P}_{\text {rot }}}{P_{\text {rot }}}$

Using the result above for $\dot{P}_{\text {rot }}$, we obtain:

$\frac{\dot{R}}{R} \simeq(-8.9 \pm 0.2) \times 10^{-12} \mathrm{~s}^{-1}$,

which is equivalent to

$\frac{\dot{R}}{R} \simeq(-2.8 \pm 0.1) \times 10^{-4} R_{\star} / \mathrm{yr}$.

If we use the radius predicted by evolutionary models for PG $1159-035$ by Kawaler \& Bradley (1994) $R_{\star}=(0.025 \pm$ $0.005) R_{\odot}$, the radius change rate $\dot{R}$ would be:

$\dot{R}=(-2.2 \pm 0.5) \times 10^{-13} R_{\odot} / \mathrm{s}$

or

$\dot{R}=(-5 \pm 1) \mathrm{km} / \mathrm{yr}$.

Using an approximation of the $517.1 \mathrm{~s}$ mode $\dot{P}$ and following the same steps, Costa et al. (1999) derived $\dot{R} / R \simeq(-4 \pm 15) \times$ $10^{-11} \mathrm{~s}^{-1}$. With the measurement of $\dot{P}$ of the $517.1 \mathrm{~s}$ mode, the obtained value for the contraction rate is far more constrained, but, as pointed out by the authors, more realistic estimations of $\dot{R} / R$ must use differential rotation at least for the outer layer of the star. Models for differentially rotating white dwarf stars calculated by Ostriker \& Bondenheimer (1968) suggest that the center rotates more rapidly than the outer layers, but not significantly so i.e. $\Omega_{\text {surface }} / \Omega_{\text {center }}>0.2$. In this case, our $\dot{R} / R$ calculated from Eq. (19) must be seen as an upper limit for the actual value:

$\left|\frac{\dot{R}}{R}\right|<-8.9 \times 10^{-12} \mathrm{~s}^{-1}$.

\subsection{The cooling rate}

We can now obtain a first estimate for the PG 1159-035 cooling rate, $\dot{T}$. The changes in period are related to two physical processes in the star: the cooling of the star and the envelope contraction (see e.g. Winget et al. 1983; Kawaler et al. 1985a):

$\frac{\dot{P}}{P} \simeq-a \frac{\dot{T}_{m}}{T_{m}}+b \frac{\dot{R}}{R}$

where $P$ is the pulsation period (for the $m=0$ multiplet component), $T_{m}$ is the temperature at the region where the model's weight function has maximum weight (where we emphasize that $T_{m}$ is the temperature, not the time of maximum, $T_{\max }$ ), $R$ is the stellar radius, and $\dot{P}, \dot{T}_{m}$, and $\dot{R}$, are the respective temporal variation rates. The constants $a$ and $b$ are positive numbers and, roughly, $a \simeq 1 / 2$ and $b \simeq 1$ (Kawaler et al. 1985b). Then,

$\frac{\dot{T}_{m}}{T_{m}}=2\left(-\frac{\dot{P}}{P}+\frac{\dot{R}}{R}\right)$.

Using $\dot{P} / P=+2.94 \times 10^{-13} \mathrm{~s}^{-1}$ for the $(m=0) 517.0 \mathrm{~s}$ pulsation mode, we obtain

$\frac{\dot{T}_{m}}{T_{m}}=(-1.84 \pm 0.04) \times 10^{-11} \mathrm{~s}^{-1}$.

Since $\dot{R} / R=0.89 \times 10^{-11} \mathrm{~s}^{-1}$, Eq. (25) implies that the PG 1159 035 temporal change in period is controlled far more by the stellar cooling than by the contraction of its envelope, as expected. The evolution of white dwarf and pre-white dwarf stars is dominated by cooling, but in hot pre-white dwarfs, the envelope contraction is still significant.

In bright pulsators such as PG 1159-035 $\left(\log \left(L / L_{\odot}\right) \simeq 2.6\right)$, the weight function reaches a maximum closer to the core of the star (Kawaler et al. 1985b). If we assume $T_{m} \simeq T_{\text {core }} \simeq 7.72 \times$ $10^{7} \mathrm{~K}$ (Alejandro Córsico, personal communication),

$\dot{T}_{m} \simeq-1.42 \times 10^{-3} \mathrm{~K} / \mathrm{s} \simeq-45000 \mathrm{~K} / \mathrm{yr}$.

\section{Summary and discussion}

The main results of our present work are:

1. The PG 1159-035 pulsation periods vary at rates of between 0.5 and $1.0 \mathrm{~ms} /$ years. For the WET data sets, the accuracy in the period determination is $10 \mathrm{~ms}$ for the least accurate cases (low amplitudes) and $1 \mathrm{~ms}$ for the most accurate ones, which enables the direct measurement of the pulsation periods after a few years of observation. After 19 years of observations, the $\dot{P}$ of the 516.0 s mode (one of the modes with higher amplitude) was measured with a relative standard uncertainty $\left(\sigma_{\dot{P}} /|\dot{P}|\right)$ of $2 \%$ and its $\ddot{P}$ could be estimated with relative standard uncertainty of $10 \%$.

2. The $\dot{P}$ of the 27 periods present in three or more yearly Fourier transforms were measured directly and the values were refined using $(\mathrm{O}-\mathrm{C})$ fitting. For seven of the pulsation modes, we used third-order $(\mathrm{O}-\mathrm{C})$ fitting to calculate $\ddot{P}$. The measured $|\ddot{P}|$ are between $1.4 \times 10^{-21} \mathrm{ss}^{-2}$ and $8.7 \times 10^{-19} \mathrm{ss}^{-2}$.

3. Using the $\dot{P}$ of the $m=0$ and $m=+1$ components of the $517.1 \mathrm{~s}$ multiplet, we estimated the rotation period change to be $\dot{P}_{\text {rot }}=(-2.13 \pm 0.05) \times 10^{-6} \mathrm{ss}^{-1}$ or $67.2 \mathrm{~s} / \mathrm{yr}$.

4. From the $\ddot{P}$ of the same multiplet, we estimated that the second order variation in the rotational period was $\ddot{P}=$ $(+9.0 \pm 0.3) \times 10^{-14} \mathrm{ss}^{-2}$. 
5. If we used the calculated values for $\dot{P}_{\text {rot }}$, the PG 1159-035 contraction rate was $\dot{R} \simeq(-5 \pm 1) \mathrm{km} \mathrm{s}^{-1}$ for $R_{\star} \simeq(0.025 \pm$ $0.005) R_{\odot}$, assuming uniform rotation, and the cooling rate is $\dot{T}_{m} \simeq-45000 \mathrm{~K} / \mathrm{yr}$, assuming $T_{m} \simeq T_{\text {core }} \simeq 7.72 \times 10^{7} \mathrm{~K}$.

Our results demonstrate that some periods decrease, while others increase, at different rates, even for the same multiplet components. Evolutionary models calculated by Kawaler \& Bradley (1994) and the La Plata group (Córsico et al. 2008; Althaus et al. 2008) predict that the $\dot{P}$ of $(\ell=1$ and $m=0)$ pulsation modes must have different values and signs (some increase, while other decrease) due to the trapping of pulsation modes in different layers of the star, but the calculated values do not fit the ob-

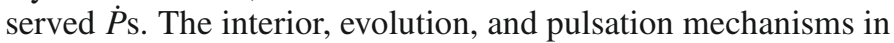
PG 1159 stars are not well known. New models have been developed by the La Plata group to fit the observed results and will be published in a future article.

A negative value for $\dot{P}_{\text {rot }}$ is expected, because pre-white dwarf stars such as PG 1159-035 undergo rapid envelope contraction processes. With contraction, the stellar radius decreases. To conserve angular momentum, the angular rotation speed must increase. In other words, its rotation period decreases, and therefore $\dot{P}_{\text {rot }}<0$. The shorter rotation periods observed in white dwarfs are of the order of few hours. This is the case, for example, for the DBV EC20058-5234 ( $P_{\text {rot }} \simeq 2 \mathrm{~h}$, Sullivan et al. 2008) and of the magnetic GD $356\left(P_{\text {rot }}=2.6 \mathrm{~h}\right.$, Brinkworth et al. 2004). If the PG 1159-035 rotation period changed from its current value to $P_{\text {rot }}=2 \mathrm{~h}$ during the DOV phase $\left(\sim 10^{6} \mathrm{yr}\right)$, the variation rate would be $\dot{P}_{\text {rot }} \simeq-0.1 \mathrm{~s} / \mathrm{yr}$. However, the calculations in Sect. 4.1 estimate that the rotation period is changing at a $\dot{P}_{\text {rot }} \simeq(-67 \pm 2) \mathrm{s} /$ year rate, far higher than expected and $\ddot{P}_{\text {rot }}$ is too excessively high to be physically acceptable. A possible explanation is that the second order effects of the stellar rotation over the rotational splitting are non-negligible as assumed in Eq. (9). The simplification of assuming a uniform rotation is also an error source in the $\dot{P}_{\text {rot }}$ calculation, but the difference in the final result must not be larger than a factor of 10 (Ostriker \& Bodenheimer 1968). On the other hand, a high de/acceleration of the stellar rotation is expected if the star recently experienced a late thermal pulse (due to ejection of matter from the star) and this may be the case for PG $1159-035$. We note that if $\left|\dot{P}_{\text {rot }}\right|$ is overestimated, both the contraction rate $|\dot{R}|$ and the cooling rate $|\dot{T}|$ must also be overestimated.

The accuracy in the determination of $\dot{P}_{\text {rot }}$ by Eq. (14) depends strongly on the accuracies of $\dot{P}_{m}$ and $\dot{P}_{0}$, far more than on the period accuracies.
Although these first results are not fully understood, we have shown that several long-term campaigns appear to be reaching sufficient accuracy to interpret evolutionary changes. The last PG 1159-035 observational campaigns were carried out in 2002 by WET. New observations of PG 1159-035 in future years should allow the direct measurement of additional pulsation modes $\dot{P}$ and $\ddot{P}$ and the refining of derived values to date, improving the calculation of the PG 1159-035 evolutionary rates.

Acknowledgements. This work was partially supported by $\mathrm{CNPq}-\mathrm{Brazil}$. We thanks Alejandro Córsico for the calculation of the PG 1159-035 $T_{\text {core }}$.

\section{References}

Althaus, L. G., Córsico, A. H., Miller Bertolami, M. M., García-Berro, E., \& Kepler, S. O. 2008, ApJ, 677, 35

Brickhill, A. J. 1975, MNRAS, 170, 407

Brinkworth, C. S., Burleigh, M. R., Wynn, G. A., \& Marsh, T. R. 2004, MNRAS, 348,33

Córsico, A. H., Althaus, L. G., Kepler,S. O., Costa, J. E. S., \& Miller Bertolami, M. M. 2008, A\&A, 478, 869

Costa, J. E. S., Kepler, S. O., \& Winget, D. E. 1995, Balt. Ast., 4, 334

Costa, J. E. S., Kepler, S. O., \& Winget, D. E. 1999, ApJ, 522, 973

Costa, J. E. S., \& Kepler, S. O. 2000, Balt. Ast., 9, 451

Costa, J. E. S., Kepler, S. O., Winget, D. E., et al. 2008, A\&A, 477, 627

Dreizler, S., \& Heber, U. 1998, A\&A, 334, 618

Hansen, C. J., Cox, J. P., \& van Horn, H. M. 1977, ApJ, 217, 151

Jahn, D., Rauch, T., Reiff, E., et al. 2007, A\&A, 462, 281

Jones, P. W., Hansen, C. J., Pesnell, W. D., \& Kawaler, S. D. 1989, ApJ, 336, 403

Kawaler, S. D. 1986, Ph.D. Thesis, Texas Univ., Austin

Kawaler, S. D., \& Bradley, P. A. 1994, ApJ, 427, 415

Kawaler, S. D., Winget, D. E., \& Hansen, C. J. 1985a, ApJ, 298, 752

Kawaler, S. D., Hansen, C. J., \& Winget, D. E. 1985b, ApJ, 295, 547

Kepler, S. O. 1993, Balt. Ast., 2, 515

Kepler, S. O., Costa, J. E. S., Mukadam, A., et al. 2005a, in 14th European Workshop on White Dwarfs, Proceedings of a meeting held at Kiel, July 19-23, 2004, ed. D. Koester, \& S. Moehler (San Francisco: ASP), ASP Conf. Ser., 334, 501

Kepler, S. O., Costa, J. E. S., Castanheira, B. G., et al. 2005b, ApJ, 634, 1311

McGraw, J. T., Starrfield, S. G., Liebert, J., \& Green, R. F. 1979, in White dwarfs and variable degenerate stars (NY: Rochester), 377

Mukadam, A. S., Kepler, S. O., Winget, D. E., et al. 2003, ApJ, 594, 961

Nather, R. E., Winget, D. E., Clemens, J. C., Hansen, C. J., \& Hine, B. P. 1990, ApJ, 361, 309

Ostriker, J. P., \& Bondenheimer, P. 1968, ApJ, 151, 1089

Sullivan, D. J., Metcalfe, T. S., O’Donoghue, D., et al. 2008, MNRAS, 556

Werner, K., Heber, U., \& Hunger, K. 1991, A\&A, 244, 437

Winget, D.E., Hanser, C. J., \& Van Horn, H. M. 1983, Nature, 303, 781

Winget, D.E., Kepler, S.O., Robinson, E.L., \& Nather, R.E. 1985, ApJ, 292, 606

Winget, D. E., Nather, R. E., Clemens, J. C., et al. 1991, ApJ, 378, 326 (W91) 